\title{
FDTD Modeling of double ultrashort pulse propagation in nonlinear absorbing media.
}

\author{
J.D. Pisonero, O. Varela, E. García, I. Hernández, J. Ajates, J.L. Sagredo, M. Olivar, C. Albarran, L. Roso and C. Méndez \\ Centro de Láseres pulsados (CLPU), Parque Científico. C/Adaja, 8. 37185 Villamayor, Salamanca, Spain.
}

\begin{abstract}
An approach based on the finite-difference time-domain (FDTD) method is developed for simulating the dynamics of two ultrashort laser pulses inside a saturable absorbing media. This work discusses the results obtained using this numerical model for the prediction of the nonlinear absorbing media behaviour as well as how it affects the final double pulse combination. These results can be used to improve contrast cleaning conditions for high power laser chains and for synchronization studies, this last application was checked in the VEGA facility lab as a code validation.
\end{abstract}

\section{Introduction}

Since the FDTD algorithm was first developed by Yee in 1966 [1], this method has been successfully applied to a large number of electromagnetic problems. In particular, an explicit scheme of this method was applied to the analysis of wave propagation in saturable absorbers and gain lasers [2]. These works showed that for small signal intensities and slowly varying pulses, the method was able to give same results as frequency-domain methods using the linear susceptibility function.

On the other hand, saturable absorbers are elements that are now included in front-end parts of high-intensity laser chains in order to enhance the temporal contrast of these laser pulses for laser-matter interaction experiments $[3,4]$.

Modelling short laser pulse propagation in these kind of materials is therefore an interesting topic to improve ultrashort pulse characteristics and management. In particular, we present results corresponding to the behaviour of two laser pulses interactions in these kind of media.

\subsection{Simulations}

\subsubsection{FDTD formulation}

A detailed description of the used method can be found in [2], where atomic rate equations describing the time evolution of atomic energy level populations under the influence of applied signals is included. In this work, the formulation used all over that reference is followed.

\subsubsection{FDTD results.}

The code was used to analyze the behaviour of two equal ultrashort laser pulses travelling inside the saturable absorber RG850. The data used to model this media are the same ones used at reference [2]. The steps values of the mesh were chosen to be $\Delta t=0.018 \mathrm{fs}$ and $\Delta z=0.002 \mu \mathrm{m}$. The resonance frequency was taken to be $\omega_{a}=2.38 \mathrm{fs}^{-1}$ and the total decay rate $\Delta \omega_{a}=0.025 \mathrm{fs}^{-1}$. Finally, the lifetime of the atoms was chosen to be $\tau_{21}=10 \mathrm{~ns}$ so the value of the factor $\kappa$ was $\kappa=7.89 \times 10^{-8}$.

Different simulations were done in order to see how one pulse affects the other depending on their initial time when both have an electric field value close to but lower than the one that is needed to saturate the absorber $\left(E_{0}=8 \mathrm{~V} / \mu \mathrm{m}\right)$. Main results are shown in Figure 1.

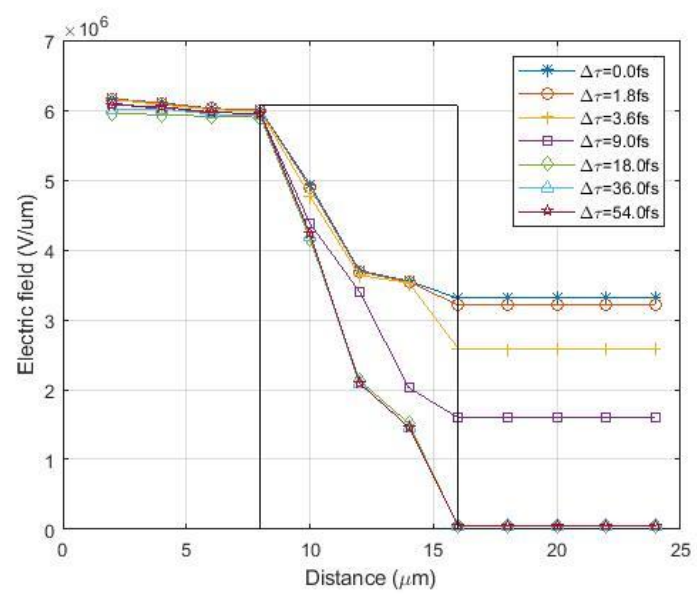

Fig. 1. Electric field during the propagation of two pulses through a SA at different delay times between pulses. SA is shown as a solid square in the graphic.

In figure 1, both pulses arrive to the SA front face after 7 microns propagation. SA width is chosen to be 8 microns. We can see that when delay between two pulses is larger than $18 \mathrm{fs}$, them both are completely absorbed in the media but the closer they become, the larger the pulse that is leaving the absorber results.

\footnotetext{
*Corresponding author: jdpisonero@clpu.es
} 


\section{Experimental results}

VEGA system is a petawatt (PW) laser user facility belonging to the Centro de Láseres Pulsados in Salamanca that allows developing experiments for exploring the physics of intense lasers interactions with matter. The Ti:Sapphire Chirped Pulse Amplification (CPA) based laser chain has three common front-end outputs (VEGA 1-2-3) at $1 \mathrm{PW}, 150 \mathrm{TW}$ and $20 \mathrm{TW}$ respectively.

Preliminary experimental validation of the FDTD code was done in the VEGA facility lab with a leak of VEGA 2 beamline (5 J, $30 \mathrm{fs}, 10 \mathrm{~Hz}$ ). The leak from VEGA2 is introduced in a Michelson interferometer that divides the initial pulse in two beams with the same energy. One of the mirror is connected to a linear stage to change the delay between both beams. After that both pulses pass through the SA and their spectrum is measured to analyze the absorption in the media.

\subsection{Results}

To analyze the results, spectrums were taken and integrated in a spectrometer at different delays between pulses. Those data are shown in Figure 2 in which there are plotted the measures of both pulses taken independently and both pulses passing together through the SA. In this figure data show that the addition of pulses spectrum taken individually is not matching with the spectrum taken with both pulses arriving at the same time, showing a quite larger transmission in this last case-

This results match with the theoretical simulations shown in Figure 3. In this figure, theoretical and experimental measurements are shown at different delays. The experimental data_were taken using a separation of 10 microns between them, what is equivalent to $33 \mathrm{fs}$ of delay. On the other hand, simulations were made with more resolution specially when the pulses are very close to each other.

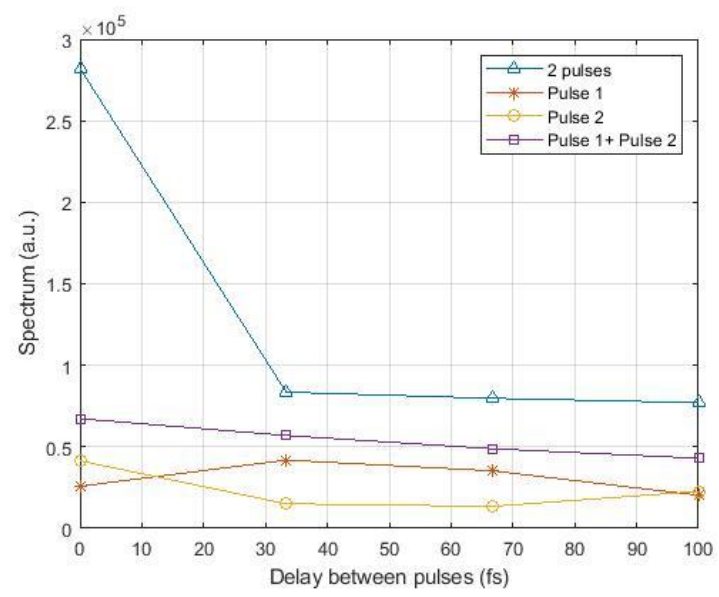

Fig. 2. Measurements of integrated spectrums with SA.

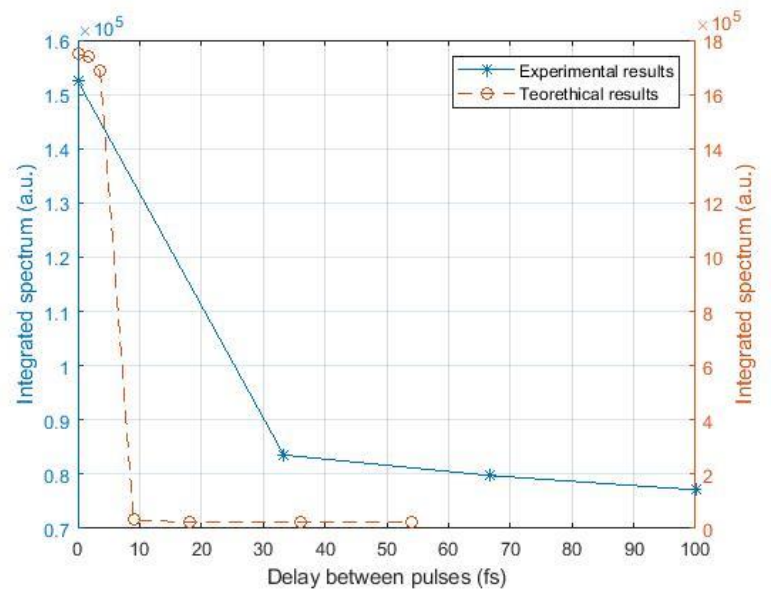

Fig. 3. Comparative between theoretical and experimental results for integrated output spectrum at different delay conditions.

The data shown in those figures prove the difference between the cases in which the pulses pass through the SA with 0 delay and those with more delay, so the less delay the pulses have, the more spectrum is measured at the output. This result is the same result obtained with the FDTD simulation.

\section{Conclusions}

In this work we have tested an implementation of an FDTD code. Simulations of pulses propagation in saturable absorbers have been developed and tested in the lab. This tool will allow future studies for short pulses synchronization with fs resolution as well as high power laser pulses contrast designs.

\section{References}

1. K.S. Yee, IEEE Trans. Antenn. Propag. 14(3), 302307 (1966)

2. Amit S. Nagra and Robert A. York, IEEE Trans. Antenn. Propag. 46, 3 (1998)

3. Jae Hee Sung, Seong Ku Lee, Tae Moon Jeong, Chang Hee Nam, Appl. Phys. B (2014) 116:287-292

4. S. Fourmaux et alt. Optics Express 19, 9, 8486 (2001) 